\title{
Optimization of goal targeting in 1 on 1 soccer robots using safe points (Experimental Research)
}

\author{
Yahya Hassanzadeh-Nazarabadi ${ }^{1}$, Abolfazl Saravani ${ }^{1}$, Bahareh Alizadeh ${ }^{2}$ \\ ${ }^{\mathbf{1}}$ Ferdowsi University, Park Sq, Mashhad, IRAN \\ ${ }^{2}$ Khayam University, Ghasem Abad, Mashhad, IRAN
}

\section{Email address:}

ya_ha_na@ieee.org(Y.H. Nazarabadi), abolfazl.saravani70@gmail.com(A. Saravani), bahareh.alzdh@gmail.com(B. Alizadeh)

\section{To cite this article:}

Yahya Hassanzadeh-Nazarabadi, Abolfazl Saravani, Bahareh Alizadeh. Optimization of Goal Targeting in 1 on 1 Soccer Robots Using Safe Points (Experimental Research).Automation, Control and Intelligent Systems. Vol. 1, No. 3, 2013, pp. 71-74.

doi: 10.11648/j.acis.20130103.16

\begin{abstract}
Due to the fact that targeting the goal is the last part of soccer robot's aggressive decision chains, aiming styles and their lack of proficiency are discussed. Then targeting based on safe points is explained and implemented. Accordingly, this method has perfect accuracy. It brings new standpoint in aiming and it's capable of moving the barrier of its related knowledge.
\end{abstract}

Keywords: 1 on 1 Soccer Robots, Digital Compass, Binary Tree, Traversing a Binary Tree

\section{Introduction}

Main goal of robocup is to create a robot team fully able to compete against world's best human soccer players until year 2050 [1]. In trying to achieve this purpose, a lot of robots has been made. For instance: Humanoid robots, Small and Middle size robots, 1 to 1 and 2 to 2 Soccer robots.

Humanoid robots' objective is to maintain balance and move like humans. There are agents inside small robots that give orders to others and they control the game. Intentions of the Middle sized ones are single processing and efficiency of their algorithms. In 1 to 1 and 2 to 2, purpose is to go beyond the individual skills to the group skills [2]. Again, the most important responsibility of robots is to score, as a result, many different optimizations have been introduced. Ideally, 1 to 1 model is considered. These intelligent robots play in a dynamic and bounded environment while tracking a special light-emitting ball.

In this paper, goal targeting optimization in 1 to 1 is discussed. First, the problem is defined in details. Then, previous works are studied and their pros and cons are explained using experimental researches with ready samples. In the next part, design of the algorithm is addressed based on artificial intelligence. In this optimization method, a hypothesis named 'safe points' is suggested and its accuracy is analyzed. In the end, conclusion and results are presented.

\section{Problem Definition}

In this section, properties of the robot, the environment in which it works and the ball are discussed. As illustrated in figure 1 , the field has $122 \mathrm{~cm}$ in width and $183 \mathrm{~cm}$ in length. Corners are flattened. Team's goals are located at the center of width of the filed at each side. They are $45 \mathrm{~cm}$ in length and $14 \mathrm{~cm}$ in height. There are cross-bars at the top of each goal to prevent robots from entering it. Its floor, walls and edges are colored. One side is blue and the other side is yellow also all of other parts of the filed are black. The ground should be positioned in a way that influence of external infrared light is as low as possible and earth magnetic territory is not disturbed in 2 meter depth. Guaranteeing the best conditions is impossible [1,2].

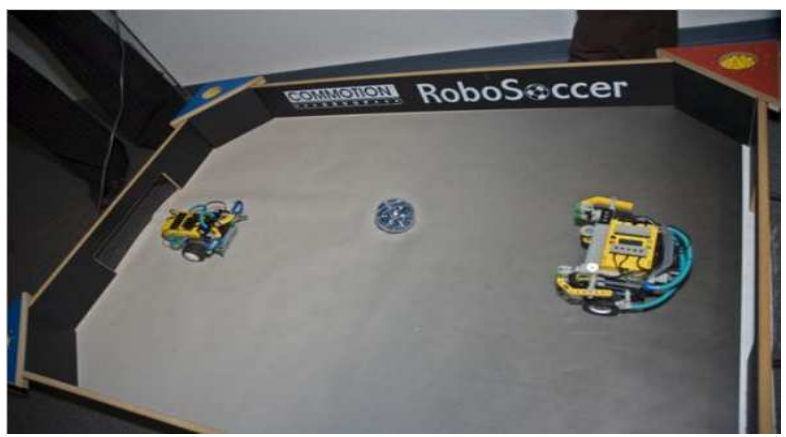

Figure1. 1 to 1 robot game field 
As exhibited in figure 2, 1 to 1 robots are sagacious. They obey specific rules for dimensions and design. These rules are edited by robocup federation every year. By standard, the robot usually can be placed in a circle with a $10 \mathrm{~cm}$ diameter $[1,2]$. Different sensors are installed on the robot. For instance, ultra sound sensor to detect surrounding walls [4] and infrared sensor to detect the ball [5]. There is also a compass attached to it, in order to indicate the angle [3]. An encoder is built in its wheel for positioning. Robot doesn't have any external power source rather than a battery, which it carries $[1,2]$. Thus all of decisions are made by the robot itself without any interference of any human being.

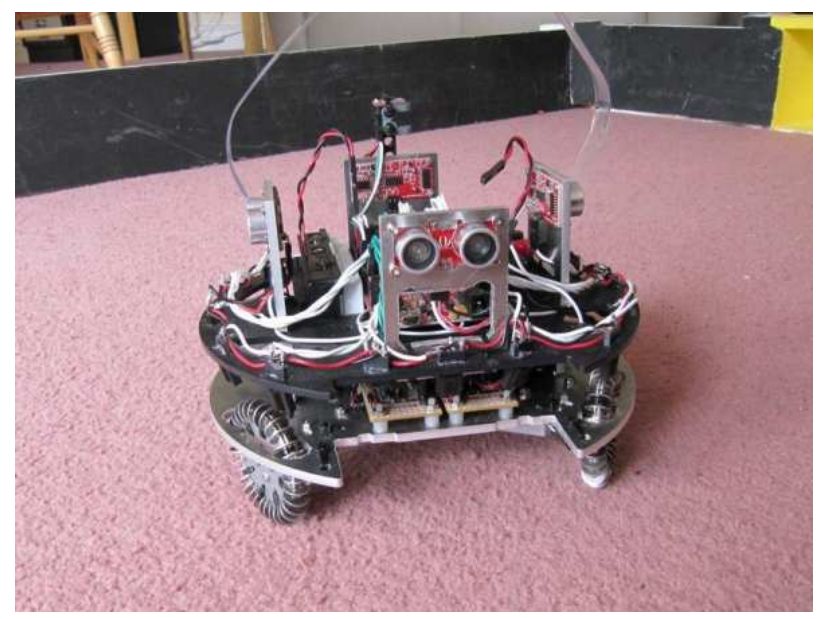

Figure 2. 1 to 1 soccer robot

Game ball is an electronic ball which casts out infrared light (IR). In this experiment, functionality of 1 to 1 robot is studied, while assuming one rival robot on the opposite side. Operation of robot is as follows: first it's situated in the field, next it finds the ball using infrared-sensor then it approaches the ball. Ball is obtained by special algorithms. Then it evaluates its next move. In the end, utilizing specific methods, goal is marked and finally it shoots.

\section{Study of Previous Work}

\subsection{Targeting the Goal Using Random Aim Locating}

This method is one the most common solution in 1 to 1 and 2 to 2 soccer robots. In thistechnique using confine of opponent's goal, a scale is computed as target. In the moment of the attack, an arbitrary angel, which is in the range of the opponent's goal is chosen by the robot and it sets its shooting system by that angle $[4,5,6]$.

Limit of the goal is calculated by the user and it is available to be utilized by algorithms of the robot. It's obvious that processing load is low, due to the fact that user gives the information to the robot. It only chooses a number from the specified range and really doesn't do any particular process at all.

Vital property of this method is haphazard selection of the angle of the objective. This random picking advances the process haste of the robot. Although, quantity of this improvement is scaled by traits of the hardware, implemented data structures and data processing algorithms. Hence, specific measurement is not possible. What is certain is 2 times gain in speed [4].

Granting the haste, random selection decreases accuracy. Because different points of the goal have different fitness values.Witnessed examination of videos of the matches shows center of the goal is more important in 1 to 1 robots and choosing it as aim point gives $98.2 \%$ precision in scoring. While for example, points with 2 centimeter distance of pole (boundary between the goal and the rest of the field) of the penalty area have lowest amount of possibility of success, which is about $31.33 \%$. Considering the presented facts, reported accuracy of this method is $41 \%$ in average. An optimist approach is 'half of the shots are made through'. Since shooting technique is vital, the amount of correctness is lower than a proper and trusted threshold. Also this method do all of its work without bearing in mind that any opponent can be in the field, which in most cases results in creating conflicts with correctness of the calculated average. For the reason, that opponent is at free will and it can have any kind of movement after shooting, it prevents targeting most of the time.

\subsection{Targeting the Goal Using Weighted Random Aim Locating}

This method is very similar to the previous technique (3-1), the only difference is that choosing the aim point from the range of possible points of the opponent's goal is adjusted constantly by multiplying it in a coefficient. This factor is 1 at the beginning of the game but it changes according to the points, in which shooting was successful. In other words, range of targeting is the whole goal at the beginning of the game and it gets smaller to the part, where chance of making aneffective shot is higher. But random basis of this method is still in effect, variety from, which target point is chosen and consequently target aim is selected is altering [7].

One of the biggest advantages of this method is high accuracy. This technique is capable of having $79.81 \%$ correctness, but it's not practical. Because robot has to be aware whether its shot is successful or not. The stated accuracy is measured, while assuming a user notifies the robot about the result of its shot. But, according to the rules of the robocup, robot has to be independent thus makes user involvement impossible. Implementing an automatic system to recognize the outcome of every shoot produces a big process load, which results in massive reduction in procedure hast. Although this technique has high amount of non-practical accuracy, but it'sunable to find a solution regarding the existence of the opponent and it's completely ignored by this method, which causes in lower correctness of $52.03 \%$.

\subsection{Targeting the Goal Using Optimized Angle}

In this method, robot is considered to be alone and there 
isn't any opponent. After it's situated inside the field, it finds the position of the ball then it's obtained by the robot. So as to target, robot's position is found by calculating the distance from walls of the field making use of ultra-sound sensor. In the end, using mathematical computation accurate angle is determined. At first, robot goes to zero angle then it turns as much as the specified angle and it shoots.

Some of the pros of this algorithm:

- $\quad$ Simple method: presented algorithm doesn't have any specific status and the computations are simple.

- Fast according to time complexity: linear time complexity

- Independent of any special hardware: there isn't any particular hardware needed. It's common to use ultra-sound sensors for determining the distance from the wall and compass for positioning.

Main defect of this method is reduced accuracy when there is an opponent. It's caused by the fact that the robot is assumed to be alone. If any rival is entered into the game, precision is reduced drastically because situating and determining the distance are interfered by rival robot. As a result, wrong angle is calculated. This matter lessens the accuracy nearly to zero percent.

Average practical precision of this method while assuming there isn't any opponent in the field is reported $100 \%$. But, accuracy is reduced to $19.02 \%$ by existence of any opponent consequently, make this method less likely to be used.

\section{Targeting the Goal Using Safe Points}

Corresponding to theprevious experiments, targeting the goal using the optimized angle while assuming the robot to be alone is an efficient method. But any interference from an opponent reduces its accuracy to almost zero percent.

To recognize the existence of the opponent, ultra-sound sensor is utilized. While maintaining zero angles, values of front and back distances are summed. The same happens to left and right gap. If these numbers are equal to length and width of the field respectively, it issues the fact that there is no opponent going to conflict in shooting otherwise preventer is identified.

To solve this problem points, located near to the robot, are used. Before approaching these point using targeting the goal method which utilizes the optimized angle,all of the related calculations are done. After reaching them, the ball is shot. From now on these points are called 'safe points'.

The key to the problem base on safe points is saving the changes of behavior of the opponent (regarding the environment) in a data structure. Afterwards velocity, acceleration andmovement equation of the opponent is worked out. Considering this information, its next move to reach the safe point is settled by binary trees traversal algorithms.

Figure 3 illustrates an example in which using safe points method is possible and figure 4 demonstrates decision making tree considering mentioned example of figure 3 .

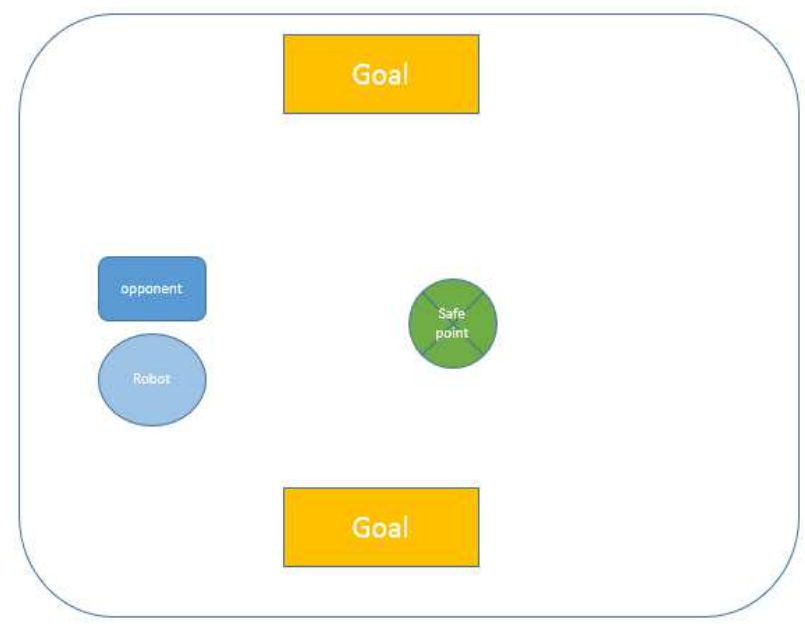

Figure 3. Positioning opponent and robot

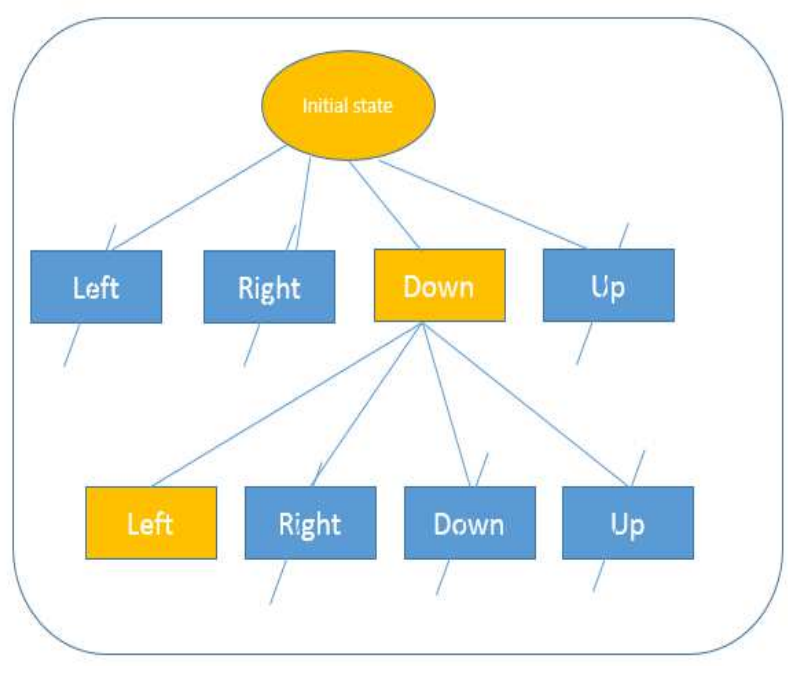

Figure 4.Decision making tree

As it can be seen from figure 4 robot can choose 4 different moves, up, down, left and right. At first, opponent is identified using ultra-sound sensor. Next, the wall on the left is recognized. Given the facts, safe point can't be in these 2 directions. For the beginning move, down is chosen so as to create enough space to do the calculations and enough distance from the opponent. After doing so, again 4 different choices appear. To prevent iteration, 'Up' move is not chosen. 'Left' move is not chosen either because of the wall. Although 'Right' and 'Left' have the same amount of computation and same distance from the opponent, 'Right' is selected owing to have less gap to the goal. At every status all of these considerations are made to pick the next move. Search operation is done as soon as robot reaches the safe point. Exclusivity of safe points is when the robot gets to them, according to analyzing the rival's behavior, targeting and shooting procedures are done before opponent reaches them. Accuracy is calculated considering the test that is explained in 'Problem Definition' section and it is shown in table 1. 
Table 1.Results of inducted experiments using safe point algorithms

\begin{tabular}{cccc}
\hline Number & X1 & Y1 & Accuracy \\
\hline 1 & 31.6 & 59.3 & $92.33 \%$ \\
2 & 42.1 & 96.7 & $89.17 \%$ \\
3 & 132.9 & 100.2 & $97.90 \%$ \\
4 & 91.6 & 90.2 & $91.32 \%$ \\
5 & 60.0 & 88.2 & $96.67 \%$ \\
\hline
\end{tabular}

In table 1, average precision is calculated using 5 points as representation of entire field. Each point is evaluated 20 times. According to technical repots of participants of Robocup 2013 and videos related to the games, accuracy of this tentative algorithm is magnificent up to this day $[4,5,6$, $7,8,9,10,11]$.

\section{Conclusion}

In this tentative experiment, 1 to 1 robots and their operational environment are presented and examined. Then, Importance of accuracy of targeting is discussed. Next, concerned practices are appraised. It ended that maximum practical accuracy of these methods is $50 \%$ which reduces drastically when existence of an opponent is considered and conflicts are created by it. In other words, all of these techniques think through the environment as a static one.

After that, targeting the goal algorithm using safe points, which is designed by the authors, is suggested. This method considers opponent making conflicts with targeting process, meaning environment is considered to be dynamic. So, aiming isn't affected by it. Average preciseness is $93.47 \%$.

\section{References}

[1] Robocup Organization, "Junior Soccer Robots Rules", Robocup, Mexico City, Mexico, 2012

[2] Iranian Robocup Organization, "Junior Soccer Robots Rules", Robocup Iran Open, Tehran, Iran, 2012

[3] Hassanzadeh Nazarabadi Y, Saghlatoon H, Sharif Shazileh A., "A method to create the most accurate goal targeting in 1 on 1 soccer robots", 5thENASE, Athens, Greece, 2010.

[4] GOROGORO, Technical Document Paper, Robocup, Mexico City, Mexico 2012.

[5] Submarine, Technical Document Paper, Robocup, Mexico City, Mexico 2012.

[6] CATASTROPHY, Technical Document Paper, Robocup, Mexico City, Mexico 2012.

[7] FRF, Technical Document Paper, Robocup, Mexico City, Mexico 2012.

[8] Fast and Smart, Technical Document Paper, Robocup, Mexico City, Mexico 2012.

[9] XYZ, Technical Document Paper, Robocup, Mexico City, Mexico 2012.

[10] FTA, Technical Document Paper, Robocup, Mexico City, Mexico 2012.

[11] A2Z, Technical Document Paper, Robocup, Mexico City, Mexico 2012. 